\title{
BMJ Global Health Effect of door-to-door distribution of HIV self-testing kits on HIV testing and antiretroviral therapy initiation: a cluster randomised trial in Malawi
}

To cite: Indravudh PP Fielding K, Chilongosi R, et al. Effect of door-to-door distribution of HIV self-testing kits on HIV testing and antiretroviral therapy initiation: a cluster randomised trial in Malawi. BMJ Global Health 2021;6:e004269. doi:10.1136/ bmjgh-2020-004269

Handling editor Seye Abimbola

- Additional material is published online only. To view, please visit the journal online (http://dx.doi.org/10.1136/ bmjgh-2020-004269).

Received 21 0ctober 2020 Revised 19 January 2021 Accepted 10 February 2021

Check for updates

(C) World Health Organization 2021. Licensee BMJ.

For numbered affiliations see end of article.

Correspondence to Pitchaya P Indravudh; pitchaya.indravudh@Ishtm. ac.uk

\section{ABSTRACT}

Introduction Reaching high coverage of HIV testing remains essential for HIV diagnosis, treatment and prevention. We evaluated the effectiveness and safety of door-to-door distribution of HIV self-testing (HIVST) kits in rural Malawi.

Methods This cluster randomised trial, conducted between September 2016 and January 2018, used restricted 1:1 randomisation to allocate 22 health facilities and their defined areas to door-to-door HIVST alongside the standard of care (SOC) or the SOC alone. The study population included residents ( $\geq 16$ years). HIVST kits were provided door-to-door by community-based distribution agents (CBDAs) for at least 12 months. The primary outcome was recent HIV testing (in the last 12 months) measured through an endline survey. Secondary outcomes were lifetime HIV testing and cumulative 16-month antiretroviral therapy (ART) initiations, which were captured at health facilities. Social harms were reported through community reporting systems. Analysis compared clusterlevel outcomes by arm.

Results Overall, 203 CBDAs distributed 273729 HIVST kits. The endline survey included 2582 participants in 11 HIVST clusters and 2908 participants in 11 SOC clusters. Recent testing was higher in the HIVST arm $(68.5 \%$ $1768 / 2582)$ than the SOC arm (48.9\%, 1422/2908), with adjusted risk difference (RD) of $16.1 \%$ (95\% $\mathrm{Cl} 6.5 \%$ to $25.7 \%$ ). Lifetime testing was also higher in the HIVST arm $(86.9 \%, 2243 / 2582)$ compared with the SOC arm $(78.5 \%$, 2283/2908; adjusted RD $6.3 \%, 95 \% \mathrm{Cl} 2.3 \%$ to $10.3 \%$ ). Differences were most pronounced for adolescents aged 16-19 years (adjusted RD 18.6\%, 95\% Cl 7.3\% to 29.9\%) and men (adjusted RD 10.2\%, 95\% Cl 3.1\% to $17.2 \%$ ). Cumulative incidence of ART initiation was 1187.2 and 909.0 per 100000 population in the HIVST and SOC arms, respectively (adjusted $\mathrm{RD} 309.1,95 \% \mathrm{Cl}-95.5$ to 713.7 ). Self-reported HIVST use was $42.5 \%$ (1097/2582), with minimal social harms reported.

Conclusion Door-to-door HIVST increased recent and lifetime testing at population level and showed high safety, underscoring potential for HIVST to contribute to HIV elimination goals in priority settings. Trial registration number NCT02718274.

\section{WHAT IS ALREADY KNOWN? \\ $\Rightarrow$ HIV self-testing (HIVST) can further extend cover- age of HIV testing among underserved population subgroups. \\ $\Rightarrow$ Limited data were previously available on the effec- tiveness and safety of HIVST from rural, underserved populations in high HIV prevalence settings. \\ WHAT ARE THE NEW FINDINGS? \\ $\Rightarrow$ Door-to-door distribution of HIVST kits by community-based distribution agents increased recent HIV testing and lifetime HIV testing, with dif- ferences most pronounced among adolescents aged 16-19 years and men. \\ $\Rightarrow$ Cumulative incidence of antiretroviral therapy ini- tiations was not shown to increase for the overall 16-month intervention period. \\ $\Rightarrow$ Self-reported HIVST use was $42.5 \%$, with minimal social harms reported. \\ WHAT DO THE NEW FINDINGS IMPLY? \\ $\Rightarrow$ Door-to-door HIVST demonstrates significant poten- tial to contribute to HIV elimination goals in priority settings.}

\section{INTRODUCTION}

In 2016, an estimated 19.4 million people were living with HIV in southern and eastern Africa. ${ }^{1}$ Despite expansion of HIV testing and treatment programmes, one-quarter of people living with HIV remained unaware of their HIV status. HIV testing gaps were highest in adolescents and men, including in Malawi. ${ }^{1}$ In 2015-2016, the proportion of undiagnosed HIV was $46 \%$ among HIV-positive adolescents and young adults aged 15-24 years, the highest across age groups. ${ }^{2}$ Of men with HIV $28 \%$ were unaware of their status compared with $20 \%$ of women with HIV. ${ }^{2}$ Reaching high coverage of HIV testing remains essential for 
HIV diagnosis, treatment and prevention, ${ }^{3}$ but access of facility-based HIV services can be limited by social, economic and health system barriers. ${ }^{4-6}$

Community-based HIV testing strategies can identify HIV-positive persons at earlier stages of infection and improve antiretroviral therapy (ART) initiation and retention when provided with universal treatment services. ${ }^{78}$ Provision of HIV self-testing (HIVST) through community-based approaches can further extend coverage of HIV testing among underserved population subgroups. ${ }^{9}$ In Malawi, urban communitybased distribution of HIVST kits achieved high uptake, with offer of home-based HIV care further increasing demand for ART. ${ }^{10}{ }^{11}$ Introducing HIVST with door-todoor HIV testing services (HTS) by community health workers increased knowledge of HIV status among urban Zambians. ${ }^{12}$ Community-based HIVST is therefore a promising approach for providing HIV testing, though lower literacy and healthcare access among rural populations could influence uptake of self-care technologies. ${ }^{13}$ Limited data were previously available on the effectiveness and safety of HIVST from rural, underserved populations in high HIV prevalence settings.

In this study, we used a cluster randomised trial to evaluate the effectiveness and safety of door-to-door distribution of HIVST kits in rural Malawi. Specifically, we aimed to assess whether distribution of HIVST kits through community-based distribution agents (CBDAs) increased the proportion of the population who tested for HIV and were initiated on ART at cluster level. Our study is part of a multicountry evaluation of community-based distribution of HIVST kits under the Unitaid/Population Services International (PSI) HIV Self-Testing Africa (STAR) Initiative.

\section{METHODS}

\section{Design, setting and participants}

We conducted a parallel cluster randomised trial of door-to-door distribution of HIVST kits. ${ }^{14}$ The study was based in 22 government primary health centres and their defined areas in four high HIV prevalence districts (Blantyre, Machinga, Mwanza, Neno). A cluster randomised design was adopted since the intervention was implemented at the health facility level. The study team enrolled health facilities providing HIV testing and ART services to rural communities in their catchment areas, with verbal consent obtained from facility representatives. Boundaries were drawn for: (1) the facility catchment area, and (2) the evaluation area within the facility catchment area. The intervention was delivered throughout the facility catchment area, while primary and secondary outcomes were measured among residents from the evaluation area. Specifically, the study population included residents aged 16 years and older from the evaluation area.

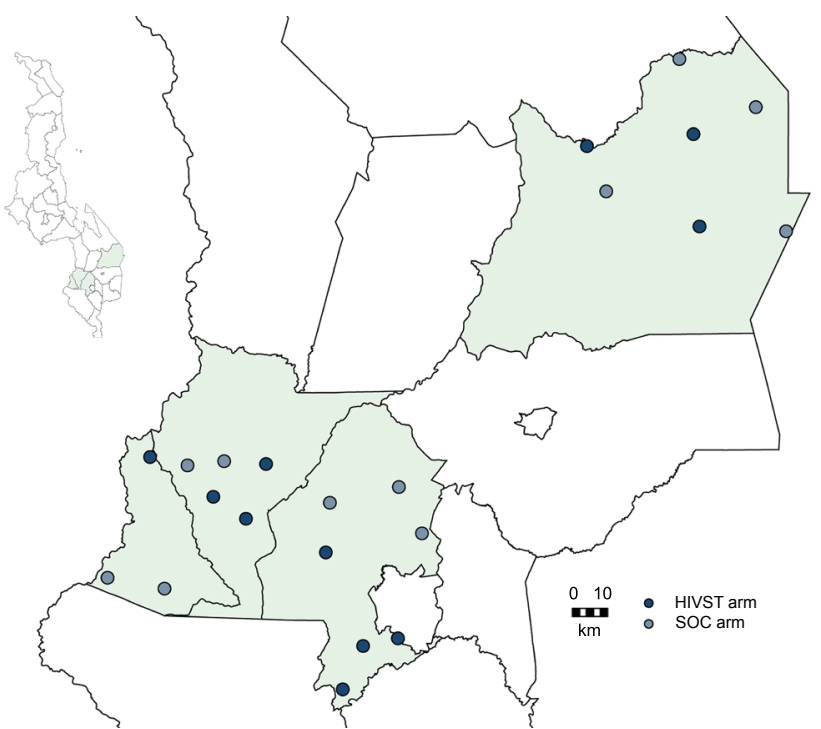

Figure 1 Map of trial clusters in Malawi. Map of Blantyre, Machinga, Mwanza and Neno district with government health facilities and their defined clusters. Malawi National Spatial Data Centre, http://www.masdap.mw/. HIVST, HIV selftesting; SOC, standard of care.

\section{Randomisation}

The 22 health facilities were randomised 1:1 to the HIVST intervention alongside the standard of care (SOC) or the SOC alone, which primarily consisted of facility-based HTS (figure 1). A computer-generated random sample was drawn by MN from 150855 unique combinations of allocating health facilities to one of the two study arms, restricted by district, catchment population size, number of HTS clients and the proportion of clients testing HIV positive. ${ }^{15}$ The final allocation was assigned at a public ceremony on 21 March 2016. Numbered balls were selected by community and government representatives from an opaque bag that corresponded to a unique allocation. Blinding of the implementation team and residents was not feasible due to the nature of the intervention, but masking was maintained where possible, including data collection, management and analysis without reference to the study arms.

A planned second randomisation of home-based HIV care in the HIVST arm was not implemented due to delays in initiating the intervention, leaving an insufficient interval for assessment. ${ }^{14}$

\section{Procedures}

The HIVST intervention was delivered for at least 12 months within the evaluation area of eligible health facilities before expanding to the rest of the facility catchment area. HIVST kits were distributed by existing CBDAs, who provided reproductive health products prior to HIVST distribution, and newly recruited CBDAs selected in consultation with village heads.

PSI Malawi conducted 1-week trainings based on an HIVST training curriculum developed in collaboration with the Ministry of Health. The training included basic information on HIV diagnosis and treatment; promoting 
HIVST using social marketing; using kits and interpreting results; providing pretest and post-test information and support, including referral for confirmatory HIV testing and ART following a positive self-test; anticipating and managing social harms; storing kits; and collecting data. National HIV testing and counselling practices and principles on voluntariness, consent and protection of client privacy and confidentiality were also covered in the training.

CBDAs then provided the OraQuick HIV Self-Test (OraSure Technologies, Thailand), along with locally adapted instructions for use, ${ }^{16}$ an opaque envelope for disposal and a self-referral card to facilitate linkage to routine HIV services at health facilities. In their respective areas, CBDAs distributed HIVST kits door-to-door or on request to residents aged 16 years and older, with their sociodemographic characteristics recorded in registers. Residents could self-test with CBDAs or in private. If residents elected to self-test privately, CBDAs followed up within 7 days of distribution to provide optional post-test support. Disclosure was not required, and HIVST results were not recorded in registers. Residents were also asked to place their used kits in envelopes to be returned to CBDAs or deposited in locked boxes located centrally in each village. PSI provided monthly supervision to verify data in CBDA registers, collect used kits and restock supplies. CBDAs were remunerated for each kit distributed (MWK100/US\$0.15) and each kit distributed with linkage to HIV care (MWK150/USD $\$ 0.23$ ).

The SOC in both arms included HIV testing and ART services under the Ministry of Health, offered primarily at health facilities. Standard HIV testing used bloodbased rapid diagnostic testing algorithms, with ART initiated immediately following a confirmed HIV diagnosis.

\section{Outcomes and measurement}

The primary outcome compared between arms the proportion of individuals aged 16 years and older who self-reported recent testing for HIV (in the last 12 months), measured at cluster level using an endline survey. Secondary outcomes compared (1) self-reported lifetime HIV testing, and (2) cumulative 16-month incidence of ART initiations per 100000 population, which was ascertained using ART clinic records during the intervention period.

HIV testing outcomes were measured through a crosssectional survey administered at the end of the intervention period. In each evaluation area, two villages with a minimum of 250 residents aged 16 years and older were randomly selected, with one village surveyed at endline and one village surveyed at baseline. The baseline survey was conducted prior to the intervention to adjust for imbalance between arms in the primary outcome.

Households in the evaluation villages were enumerated and randomly selected to provide a sample of at least 250 participants per village. All individuals aged 16 years and older in selected households were eligible for the survey, with multiple visits for interviews attempted to maximise the response rate. Informed verbal consent or assent was obtained. Participants were then interviewed on household and sociodemographic characteristics and prior use of HIV testing, treatment and prevention services.

ART initiation data were extracted from registers at each of the health facilities for the 16-month intervention period and the 12-month period preceding the intervention. Eligibility criteria included ART patients aged 16 years and older from the evaluation area. Population estimates for the evaluation area, which were used as the denominator for the ART outcome, were obtained from facility and village registers.

The proportion of lifetime HIVST use and the number of HIVST kits distributed were evaluated using the endline survey and CBDA registers. Adverse events related to HIVST were also measured using the endline survey in addition to a community reporting system established in evaluation villages to identify and manage potential adverse events. ${ }^{17}$ Community stakeholders, including village heads, community health workers, religious leaders and police officers, documented, investigated and managed social harms related to HIV testing and self-testing. Adverse events were reported to the study team and assessed, categorised by severity and followed up as appropriate. ${ }^{17}$

\section{Sample size}

With 11 clusters per arm and 250 participants per cluster, we had at least $80 \%$ power at a $5 \%$ significance level to detect a $30 \%$ relative increase in the primary outcome of recent HIV testing in the HIVST arm, assuming 25\%-40\% coverage in the SOC arm. ${ }^{18}$ The study was also powered to identify a $45 \%$ relative increase in lifetime HIV testing in the HIVST arm, assuming $42 \%-60 \%$ coverage in the SOC $\mathrm{arm}$. The sample size was calculated using a coefficient of variation $(\mathrm{k})$ in clusters of $0.25 .^{15}$

\section{Statistical analysis}

We conducted an intention-to-treat analysis based on cluster assignment to study arms and used methods appropriate for cluster randomised trials. ${ }^{15}$ The risk difference (RD) and risk ratio were calculated respectively from cluster-level risks and log risks, which were compared by arm using a t-test. For HIV testing outcomes, we adjusted for imbalances in individual-level covariates based on a two-stage approach. ${ }^{15}$ The first stage used logistic regression with individual-level covariates to obtain predicted values, which were summed at the cluster level and applied to calculate the difference and ratio of observed and predicted values. The second stage used linear regression of covariate-adjusted residuals obtained from the first stage and included the study arm. To adjust for imbalance in the primary outcome prior to the intervention, the cluster-level baseline covariate of recent HIV testing was also included in the regression model. The ART initiation outcome adjusted for ART uptake in the 12-month preintervention period. 


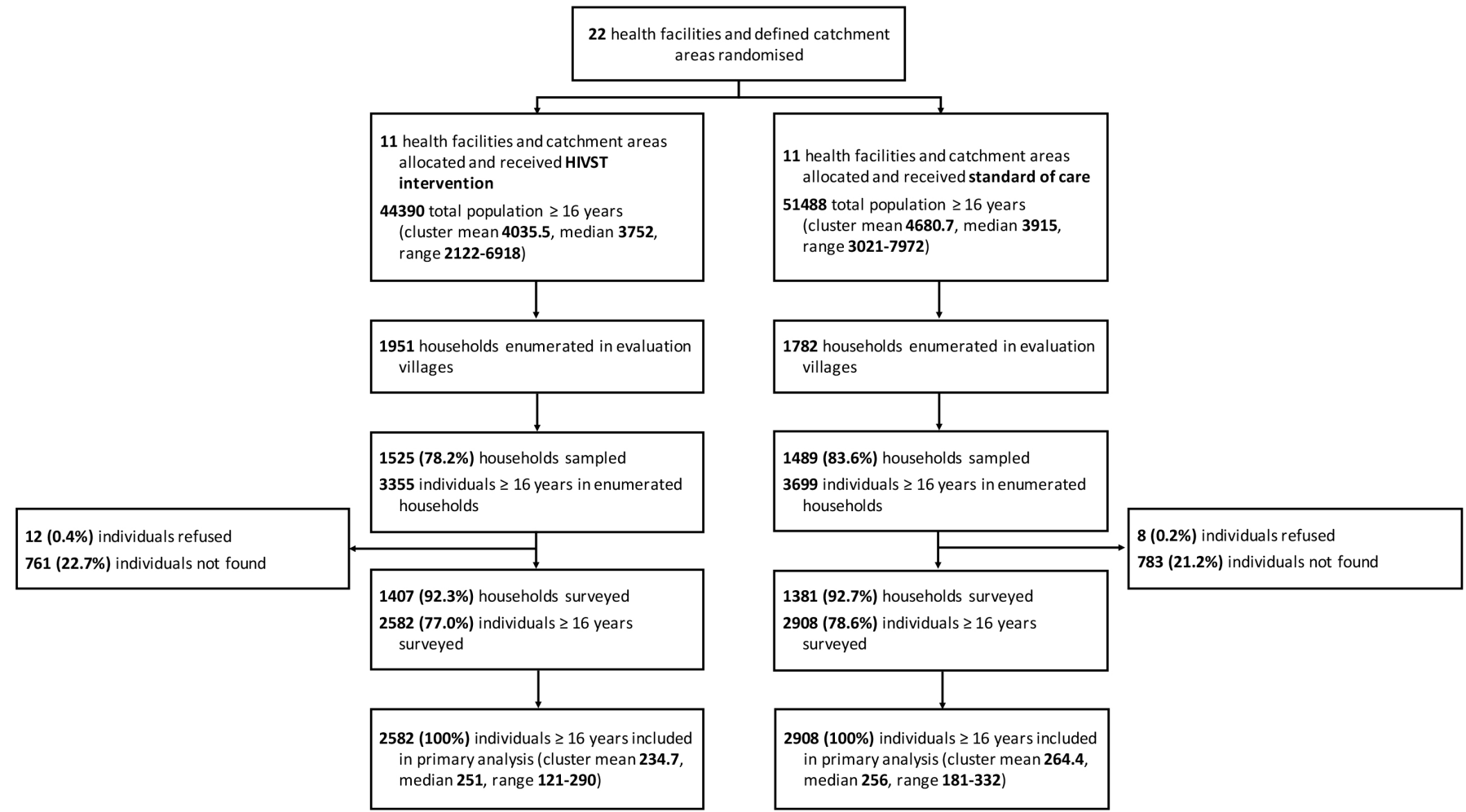

Figure 2 Trial flow diagram. Flow diagram of the cluster randomised trial. HIVST, HIV self-testing.

For recent HIV testing, a priori subgroup analyses were specified by sex, age group (16-19 years, 20 years and older) and socioeconomic status (lowest, middle, highest strata). Post hoc analysis used alternative categories of age group (16-19 years, 20-39 years, 40 years and older). Further, subgroup analyses were conducted for lifetime HIV testing by sex, age group and socioeconomic status, and for ART initiations by intervention period $(0-5,6-11$, 12-16 months). Statistical analysis used Stata V.14.0.

\section{RESULTS}

Implementation of the intervention was staggered, starting from September to December 2016, and continuing until January 2018. Overall, 203 CBDAs (cluster mean 18.5) distributed 273729 HIVST kits (cluster mean 24884.45 ) throughout the catchment area of health facilities, including evaluation and non-evaluation areas (online supplemental table 1). The volume of kits distributed was similar by sex, with $50.2 \% \quad(n=137433)$ of kits delivered to men.

The population in the evaluation area included 44 390 residents in 11 clusters in the HIVST arm and 51 488 residents in 11 clusters in the SOC arm. Participants were recruited for the endline survey between October 2017 and January 2018. The trial flow diagram is reported in figure 2. The endline survey included $77.0 \%$ (2582/3355) of enumerated residents in the HIVST arm and $78.6 \%$ (2908/3699) of enumerated residents in the SOC arm, with few eligible residents refusing to participate $(10 / 7054)$.
Population characteristics for the endline survey are summarised in table 1 . The proportion of men was $42.6 \%$ $(2339 / 5490)$ and the median age was 31 years old. The majority of participants did not have beyond primarylevel education $(84.9 \%, 4661 / 5490)$. Most characteristics were well balanced by arm. Differences were observed for marital status, with $69.5 \%(1795 / 2582)$ married in the HIVST arm and $63.2 \%(1838 / 2908)$ married in the SOC arm.

The baseline survey was administered between May and August 2016. Of listed individuals, 78.5\% (2809/3577) and $74.7 \%$ (2664/3567) were surveyed in the HIVST and SOC arms, respectively (online supplemental tables 2 and $3)$. Baseline coverage of HIV testing in the last 12 months was higher in the HIVST arm $(56.0 \%, 1574 / 2809)$ than the SOC arm $(48.4 \%, 1289 / 2664$; table 1$)$. We therefore adjusted for baseline differences in analysis of primary and secondary outcomes. Self-reported lifetime use of HIVST at baseline was limited (7/5473).

\section{Primary outcome}

At endline, recent HIV testing (in the last 12 months) was higher in the HIVST arm $(68.5 \%, 1768 / 2582)$ compared with the SOC arm $(48.9 \%, 1422 / 2908)$, with adjusted RD of $16.1 \%$ (95\% CI $6.5 \%$ to $25.7 \%$; table 2 ; online supplemental figure 1). In subgroup analysis, the intervention had a more pronounced effect among adolescents aged 16-19 years (adjusted RD $26.1 \%, 95 \%$ CI $12.9 \%$ to $39.2 \%$ ) than adults aged 20 years and older (adjusted RD 14.7\%, $95 \%$ CI $4.8 \%$ to $24.6 \%$ ), though data were consistent with no interaction effect ( $p$ value for interaction $=0.18$ ). Post 
Table 1 Comparison of endline population characteristics by study arm

\begin{tabular}{|c|c|c|}
\hline & HIVST & SOC \\
\hline & n (\%) & n (\%) \\
\hline \multicolumn{3}{|l|}{ Baseline } \\
\hline Individual characteristics & $(n=2809)$ & $(n=2664)$ \\
\hline Ever tested & $82.2 \%(2308)$ & $77.1 \%(2054)$ \\
\hline Tested in the last 12 months & $56.0 \%(1574)$ & $48.4 \%(1289)$ \\
\hline \multicolumn{3}{|l|}{ Endline } \\
\hline Household characteristics & $(n=1407)$ & $(n=1381)$ \\
\hline Adults (median/range) & $2(1-7)$ & $2(1-9)$ \\
\hline Children (median/range) & $2(0-8)$ & $2(0-9)$ \\
\hline \multicolumn{3}{|l|}{ Household wealth index* } \\
\hline Lowest & $434(32 \%)$ & 468 (35.7\%) \\
\hline Middle & $456(33.6 \%)$ & $422(32.2 \%)$ \\
\hline Highest & 466 (34.4\%) & $421(32.1 \%)$ \\
\hline Individual characteristics & $(n=2582)$ & $(n=2908)$ \\
\hline Male & $1075(41.6 \%)$ & $1264(43.5 \%)$ \\
\hline Age (median/range) & $31(16-97)$ & $31(16-91)$ \\
\hline \multicolumn{3}{|l|}{ Age group (years) } \\
\hline $16-19$ & $366(14.2 \%)$ & $439(15.1 \%)$ \\
\hline $20-24$ & $469(18.2 \%)$ & $558(19.2 \%)$ \\
\hline $25-39$ & $931(36.1 \%)$ & 998 (34.3\%) \\
\hline$\geq 40$ & $816(31.6 \%)$ & $913(31.4 \%)$ \\
\hline \multicolumn{3}{|l|}{ Marital status } \\
\hline Married or living together & $1795(69.5 \%)$ & $1838(63.2 \%)$ \\
\hline $\begin{array}{l}\text { Separated, divorced or } \\
\text { widowed }\end{array}$ & $405(15.7 \%)$ & $438(15.1 \%)$ \\
\hline Never married & $382(14.8 \%)$ & $632(21.7 \%)$ \\
\hline \multicolumn{3}{|l|}{ Educational attainment } \\
\hline None & $463(17.9 \%)$ & $555(19.1 \%)$ \\
\hline Primary & $1745(67.6 \%)$ & $1898(65.3 \%)$ \\
\hline Secondary or higher & $374(14.5 \%)$ & $455(15.6 \%)$ \\
\hline Literate & $1639(63.5 \%)$ & $1907(65.6 \%)$ \\
\hline Resident in last 12 months & $2429(94.1 \%)$ & $2748(94.5 \%)$ \\
\hline \multicolumn{3}{|l|}{ Self-rated health status $\dagger$} \\
\hline Very good & $662(25.7 \%)$ & 996 (34.3\%) \\
\hline Good & $1470(57.0 \%)$ & $1429(49.1 \%)$ \\
\hline Fair & $319(12.4 \%)$ & $320(11.0 \%)$ \\
\hline Poor & $128(5.0 \%)$ & $163(5.6 \%)$ \\
\hline
\end{tabular}

Samples for the baseline and endline survey include different individuals.

*51 missing values in the HIVST arm and 70 missing values in the SOC arm.

† 3 missing values in the HIVST arm.

HIVST, HIV self-testing; SOC, standard of care.

hoc analysis found similar differences among adults aged 20-39 years and adults 40 years and older (online supplemental table 4). While the difference in the proportion of recent testing was also higher for men (adjusted RD $20.6 \%, 95 \%$ CI $7.1 \%$ to $34.0 \%$ ) compared with women (adjusted RD $12.8 \%$, 95\% CI $4.0 \%$ to $21.6 \%$ ), there was weak evidence for interaction $(p=0.07)$. In terms of socioeconomic status, the effect of the intervention was greater among participants in the highest strata (adjusted RD $20.2 \%, 95 \%$ CI $8.3 \%$ to $32.1 \%$ ) than the lowest strata (adjusted RD $13.0 \%, 95 \%$ CI $1.1 \%$ to $24.8 \%$ ). However, there was no evidence for interaction (highest vs lowest strata: $p$ value for interaction $=0.18$ ).

\section{Secondary outcomes}

Lifetime HIV testing was higher in the HIVST arm $(86.9 \%$, 2243/2582) than the SOC arm $(78.5 \%, 2283 / 2908$; adjusted RD $6.3 \%, 95 \%$ CI $2.3 \%$ to $10.3 \%$; table 2 ; online supplemental figure 1). In exploratory analysis, the effect of the intervention was larger for adolescents aged 16-19 years (adjusted RD 18.6\%, 95\% CI $7.3 \%$ to $29.9 \%$ ) than adults aged 20 years and older (adjusted RD 4.4\%, 95\% CI $0.5 \%$ to $8.2 \%$; $\mathrm{p}$ value for interaction $=0.02$ ), and for men (adjusted RD 10.2\%, 95\% CI $3.1 \%$ to $17.2 \%$ ) than women (adjusted RD 3.4\%, 95\% CI $-0.9 \%$ to $7.7 \%$; p value for interaction $=0.07$; online supplemental table 4 ). The intervention effect on lifetime testing was similar in the lowest and highest socioeconomic strata and close to zero for the middle strata.

Cumulative incidence of ART initiation captured at health facilities during the 16-month intervention period was 1187.2 and 909.0 per 100000 population in the HIVST and SOC arms, respectively (adjusted RD 309.1, $95 \%$ CI -95.5 to 713.7 ; table 2; online supplemental figure 1). Stratified by time period since study initiation, the adjusted RD for periods of $0-5,6-11$ and $12-16$ months was 142.6 (95\% CI -81.5 to 366.7 ), 194.8 (95\% CI 31.4 to 358.1 ) and 28.3 (95\% CI -186.5 to 129.9 ), respectively. There was no evidence for statistical differences measured (6-11 months vs $0-5$ months: $p$ value for interaction $=0.51 ; 12-16$ months vs $0-5$ months: $p$ value for interaction $=0.42$; online supplemental table 4 ).

\section{Process outcomes}

Consistent with the high number of HIVST kits distributed, there were large differences between arms in awareness and use of HIVST at endline. The proportion of participants who had heard of HIVST at endline was $88.8 \%(2294 / 2582)$ in the HIVST arm and $31.5 \%$ $(917 / 2908)$ in the SOC arm (table 3). Self-reported lifetime HIVST use was respectively $42.5 \%(1097 / 2582)$ in the HIVST arm, with uptake highest in young men aged $20-24$ years $(58 \%)$ and adolescent boys $(49.0 \%$; online supplemental figure 2). Similar coverage was reported for HIVST use in the last 12 months. HIVST use was $8.3 \%$ $(240 / 2908)$ in the SOC arm, with one cluster exposed to an external community-based HIVST programme in 2017. Among participants who recently self-tested in the HIVST arm $(\mathrm{n}=794)$, most received HIVST kits from the CBDA $(97.9 \%, \mathrm{n}=777)$ and collected their kits at home $(76.7 \%, \mathrm{n}=609)$. Further, $0.8 \%(\mathrm{n}=6)$ reported a new HIV-positive result and 2.0\% ( $\mathrm{n}=16)$ reported a 


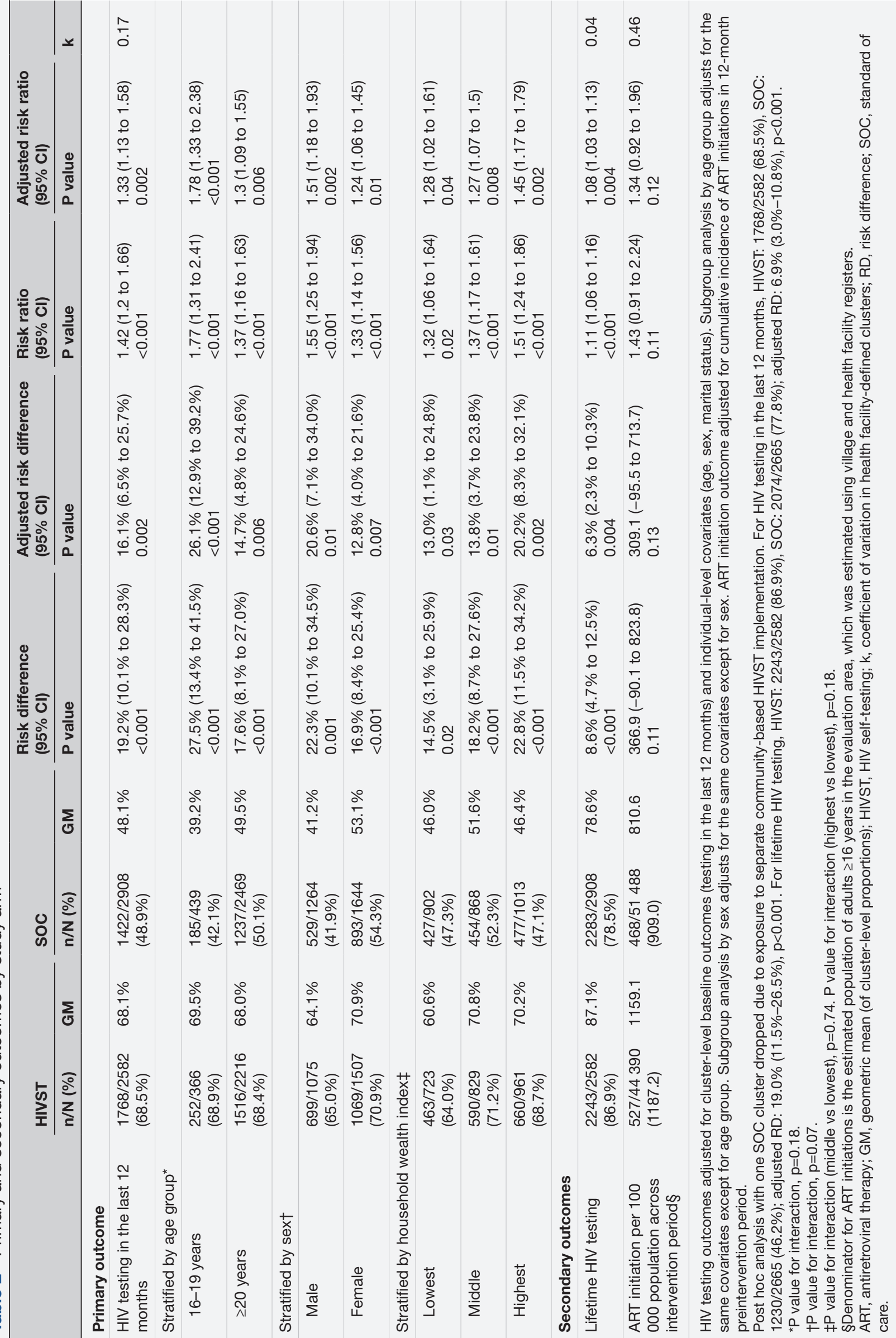


Table 3 Fidelity to HIV self-testing intervention

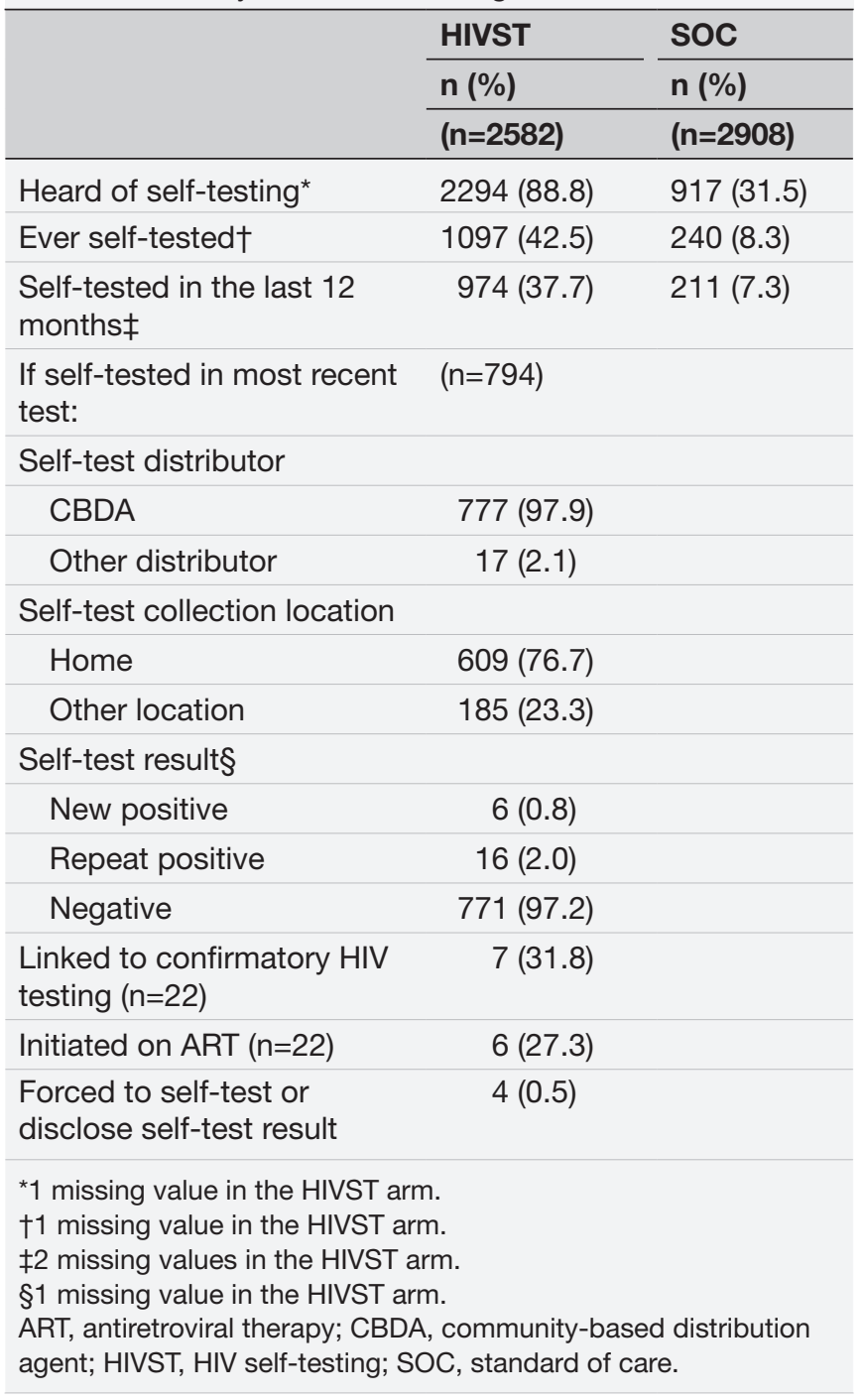

repeat positive result, of whom $31.8 \%(7 / 22)$ linked to confirmatory HIV testing and $27.3 \%(6 / 22)$ initiated on ART.

\section{Safety outcomes}

At endline, $0.5 \%$ (4/794) of participants reported being forced to self-test or disclose their self-test results (table 3 ). Three events of social harm related to HIVST were reported, managed and resolved through the community reporting system: one case involved discrimination from household members for collecting an HIVST kit; two cases involved temporary separation between couples, with one event from self-testing and one event due to newly identified serodiscordancy within the couple. In an additional event reported to implementers in nonevaluation areas, a perinatally infected adolescent under the eligible age acquired an HIVST kit and suffered a highly stigmatising response following self-testing with her friends. These events have been described in detail elsewhere. $^{17}$

\section{DISCUSSION}

The main findings from this cluster randomised trial were that door-to-door distribution of HIVST kits by CBDAs increased recent and lifetime HIV testing at population level in rural Malawi. Our primary outcome of recent testing increased by $16.1 \%$. Lifetime testing increased by $6.3 \%$, with differences between arms most pronounced among priority subgroups: adolescents aged 16-19 years and men. The HIVST intervention did not show an effect on cumulative incidence of ART initiations at health facilities for the overall 16-month intervention period. HIVST use was reported by $42.5 \%$ of participants in the HIVST arm, with uptake highest among young men aged 20-24 years and adolescent boys. Few serious adverse events were reported. Our results therefore support door-to-door HIVST as an effective and safe strategy that can be used to meet HIV testing needs in underserved rural populations.

Our study is one of three community-based randomised trials from rural settings in southern Africa that were implemented as part of STAR. ${ }^{141920}$ Affordable, convenient and safe HIV testing strategies are important for rural populations, who often have more pronounced barriers to accessing healthcare. ${ }^{13}$ The STAR trials had critical differences that can be used to guide policy and future research priorities. Our results showed increased recent and lifetime HIV testing from door-to-door HIVST, consistent with a separate Zambian trial, which added HIVST to an intensive community-based HIV programme. ${ }^{12}$ High coverage of lifetime testing $(88.7 \%)$ and lifetime HIVST use $(50.2 \%)$ was reported for both arms of the STAR Zimbabwe trial, which compared the impact of remuneration strategies under campaign-style distribution by CBDAs on linkage to HIV care. ${ }^{19}$ Our study, along with the Zimbabwe trial, implemented doorto-door distribution. In contrast, provision of HIVST kits at home, high-density community sites and health facilities under the STAR Zambia trial resulted in lower HIVST use $(26.3 \%)$ and no measurable increase in lifetime or recent testing. ${ }^{20}$ Process outcomes, such as HIVST awareness, were also lower in Zambia than in Malawi and Zimbabwe, suggesting that door-to-door distribution can lead to higher penetration than broader communitybased models. 1920

We showed encouraging uptake of HIVST, with minimal social harms reported. Uptake was highest among young men aged 20-24 years followed by adolescent boys aged 16-19 years. Our study also reported increased lifetime HIV testing among adolescents and men. HIVST can bypass barriers that prevent uptake of standard HTS by these priority subgroups, ${ }^{10} 21$ with HIVST valued for the convenience and confidentiality afforded. ${ }^{522}$ However, our results demonstrated lower uptake compared with the STAR Zimbabwe trial, which evaluated more intensive distribution across a shorter period of time. ${ }^{19}$ Similarly, a previous study in urban Malawi reported $84 \%$ uptake from distribution of HIVST kits by community volunteers, which may indicate higher acceptability 
among urban counterparts. ${ }^{10}$ Understanding remaining demand-side barriers may allow for further optimisation of community-based HIVST strategies to maximise coverage and impact among underserved subgroups. Alternative HIVST strategies should also be considered. In Malawi, facility-based provision of HIVST kits among outpatients increased coverage of HIV testing, especially among adolescents. ${ }^{23}$ Another study in Malawi found that secondary distribution to male partners of pregnant women extended testing coverage. ${ }^{21}$

Our study did not observe an increase in ART initiations for the overall 16-month intervention period but in subgroup analysis for the 6-11 months' postintervention period. Further, $0.8 \%$ of participants reported a new positive result from HIVST, with frequent repeat testing among participants already known to be HIV positive. Impact on ART uptake varied across STAR trials. ${ }^{24} \mathrm{~A}$ nonrandomised evaluation accompanying the Zimbabwe trial estimated a $27 \%$ increase in ART initiation rates, ${ }^{19}$ while no difference was observed in Zambia. ${ }^{20}$ Linkage to HIV care is practically difficult to capture, with potential for measurement errors. ${ }^{24}$ True impact on ART demand from HIVST will depend on the prevalence of untreated HIV, which has been declining in southern and eastern Africa. ${ }^{2}$ The intensity and reach of HIVST distribution strategies will also influence population-level impact. Additionally, interventions to encourage timely linkage to health facilities may be required, such as provision of home-based HIV care or more substantial financial incentives. ${ }^{11} 21$

The benefits of community-based HTS are well established, with the main barrier to implementation including high cost per test and cost per new diagnosis, especially as countries reach the 'First 90' targets. ${ }^{25}$ Economic analysis of our intervention is reported separately. ${ }^{26} \mathrm{CBDA}$ distribution showed average cost of 2017 US $\$ 8.15$ per HIVST kit distributed, with the main cost contributors including personnel and HIVST kits. ${ }^{26}$ Unit cost of communitybased HIVST was higher than the average cost of facilitybased HTS (2016 US\$4.92) and facility-based HIVST (US\$4.99) in Malawi. ${ }^{23}{ }^{27}$ While community-based HIVST is likely to maintain higher levels of knowledge of recent HIV status than standard HTS alone, sustainable provision will require further reductions in costs and optimisation of linkage to HIV treatment and prevention. For example, providing periodic campaigns is likely to be less costly than maintaining a continuous programme, especially if targeted to high-prevalence populations or underserved subgroups with ongoing HIV risk. Alternatively, a community-led approach for delivering HIVST has potential to further reduce costs. ${ }^{28}$

The main strength of this study is the use of a robust cluster randomised design to report on the effectiveness and safety of large-scale implementation of communitybased HIVST. Further, CBDAs are commonly used to distribute health commodities in Malawi, with our findings potentially generalisable to settings in sub-Saharan Africa with similar community health cadres. We also add to the body of evidence on effective strategies for expanding HIV testing coverage in rural, HIV-prevalent populations and among population subgroups with substantial undiagnosed HIV.

Limitations included HIV testing outcomes that were self-reported and therefore susceptible to misreporting. ART initiations may be underestimated if study residents accessed non-study health facilities, which we aimed to minimise with our inclusion criteria of health facilities. Non-participation in the endline survey could result in ascertainment bias, with response rates lower among men than women. We did not account for householdlevel clustering, though this was unlikely to have altered our findings. ${ }^{29}$ We discontinued second randomisation of home-based HIV care in the HIVST arm; however, the outcomes reported in this study were not affected. Data on social harms were passively collected through community reporting systems, potentially under-reporting the number of adverse events. Finally, our findings are limited to our intervention design, which included doorto-door implementation through remunerated CBDAs.

\section{CONCLUSION}

Door-to-door distribution of HIVST kits by CBDAs increased recent and lifetime HIV testing in rural, underserved populations, including among adolescents 16-19 years and men. ART initiations showed no differences between arms for the overall 16-month intervention period. HIVST was very acceptable and safe, with uptake highest among young men and adolescent boys. Doorto-door HIVST demonstrates significant potential to contribute to HIV elimination goals in priority settings. Further, as countries approach the 'First 90' targets, this approach could be adapted for periodic implementation to meet the ongoing need for HTS in settings with high undiagnosed HIV.

\section{Author affiliations \\ ${ }^{1}$ Department of Global Health and Development, Faculty of Public Health and Policy, London School of Hygiene \& Tropical Medicine, London, UK \\ ${ }^{2}$ Malawi-Liverpool-Wellcome Trust Clinical Research Programme, Blantyre, Malawi ${ }^{3}$ Department of Infectious Disease Epidemiology, Faculty of Epidemiology and Population Health, London School of Hygiene \& Tropical Medicine, London, UK ${ }^{4}$ School of Public Health, University of the Witwatersrand, Johannesburg- Braamfontein, Gauteng, South Africa \\ ${ }^{5}$ Population Services International, Blantyre, Malawi \\ ${ }^{6}$ Department of HIV and AIDS, Ministry of Health, Lilongwe, Malawi \\ ${ }^{7}$ Global HIV, Hepatitis and Sexually Transmitted Infections Programmes, World Health Organization, Geneve, Switzerland \\ ${ }^{8}$ Department of Clinical Research, Faculty of Infectious and Tropical Diseases, London School of Hygiene \& Tropical Medicine, London, UK \\ ${ }^{9}$ Department of International Public Health, Liverpool School of Tropical Medicine, Liverpool, UK \\ ${ }^{10}$ Tropical Infectious Diseases Unit, Royal Liverpool University Hospital, Liverpool, UK \\ ${ }^{11}$ Population Services International, Washington, DC, USA \\ Twitter Miriam Taegtmeyer @MiriamTaegtmeye}

Acknowledgements We are grateful to the study participants; the Blantyre, Machinga, Mwanza and Neno District Health Offices; the Department of HIV and AIDS at the Ministry of Health; the Malawi-Liverpool-Wellcome Trust Clinical 
Research Programme and Population Services International Malawi teams; and the technical advisory group.

Contributors ELC conceptualised the study. PPI, KF, RC, ND, KH and ELC contributed to the study design. PPI, MK, RC and RNz supervised the implementation of the study, including the intervention and data collection. PPI, $\mathrm{MN}$ and $\mathrm{KF}$ conducted the statistical analysis. PI, KF, RNy, CCJ, MT, ND, KH and ELC critically interpreted the results. PPI wrote the first draft of the manuscript. All authors reviewed and approved the final manuscript.

Funding The study was funded by Unitaid (grant number: P0 8477-0-600). ELC is also funded by the Wellcome Trust (WT091769).

Competing interests None declared.

Patient consent for publication Not required.

Ethics approval The trial is registered with ClinicalTrials.gov. The trial protocol (https://hivstar.lshtm.ac.uk/protocols/) was approved by the University of Malawi College of Medicine (ref: P.01/16/1861) and the London School of Hygiene \& Tropical Medicine (ref: 10566).

Provenance and peer review Not commissioned; externally peer reviewed.

Data availability statement Data are available in a public repository. Data are available upon request through datacompass.Ishtm.ac.uk. The protocol, statistical analysis plan, and CONSORT checklist are available as online supplementary files.

Supplemental material This content has been supplied by the author(s). It has not been vetted by BMJ Publishing Group Limited (BMJ) and may not have been peer-reviewed. Any opinions or recommendations discussed are solely those of the author(s) and are not endorsed by BMJ. BMJ disclaims all liability and responsibility arising from any reliance placed on the content. Where the content includes any translated material, BMJ does not warrant the accuracy and reliability of the translations (including but not limited to local regulations, clinical guidelines, terminology, drug names and drug dosages), and is not responsible for any error and/or omissions arising from translation and adaptation or otherwise.

Open access This is an open access article distributed under the terms of the Creative Commons Attribution IGO License (CC BY NC 3.0 IGO), which permits use, distribution,and reproduction in any medium, provided the original work is properly cited. In any reproduction of this article there should not be any suggestion that WHO or this article endorse any specific organization or products. The use of the WHO logo is not permitted. This notice should be preserved along with the article's original URL.

Disclaimer: The author is a staff member of the World Health Organization. The author alone is responsible for the views expressed in this publication and they do not necessarily represent the views, decisions or policies of the World Health Organization.

\section{ORCID iDs}

Pitchaya P Indravudh http://orcid.org/0000-0003-4013-7645

Melissa Neuman http://orcid.org/0000-0002-8870-6504

\section{REFERENCES}

1 UNAIDS. Unaids data 2017. Geneva, Switzerland: Joint United Nations Programme on HIV/AIDS (UNAIDS), 2017.

2 Ministry of Health [Malawi]. Malawi population-based HIV impact assessment (MPHIA) 2015-2016: final report. Lilongwe: Ministry of Health, 2018.

3 Abdool Karim SS, Karim SSA. HIV-1 Epidemic Control - Insights from Test-and-Treat Trials. N Engl J Med 2019;381:286-8.

4 Chikwari CD, Dringus S, Ferrand RA. Barriers to, and emerging strategies for, HIV testing among adolescents in sub-Saharan Africa. Curr Opin HIV AIDS 2018;13:257-64.

5 Indravudh PP, Sibanda EL, d'Elbée M, et al. 'I will choose when to test, where I want to test': investigating young people's preferences for HIV self-testing in Malawi and Zimbabwe. AIDS 2017;31 Suppl 3:S203-12.

6 Sharma M, Barnabas RV, Celum C. Community-Based strategies to strengthen men's engagement in the HIV care cascade in subSaharan Africa. PLoS Med 2017;14:e1002262.

7 Sharma M, Ying R, Tarr G, et al. Systematic review and metaanalysis of community and facility-based HIV testing to address linkage to care gaps in sub-Saharan Africa. Nature 2015;528:S77-85.
8 Havlir D, Lockman S, Ayles $\mathrm{H}$, et al. What do the universal test and treat trials tell us about the path to HIV epidemic control? J Int AIDS Soc 2020;23:e25455.

9 WHO. Consolidated guidelines on HIV testing services for a changing epidemic. Geneva: World Health Organization (WHO), 2019.

10 Choko AT, MacPherson P, Webb EL, et al. Uptake, accuracy, safety, and linkage into care over two years of promoting annual self-testing for HIV in Blantyre, Malawi: a community-based prospective study. PLoS Med 2015;12:e1001873.

11 MacPherson P, Lalloo DG, Webb EL, et al. Effect of optional home initiation of HIV care following HIV self-testing on antiretroviral therapy initiation among adults in Malawi: a randomized clinical trial. JAMA 2014;312:372-9.

12 Mulubwa C, Hensen B, Phiri MM, et al. Community based distribution of oral HIV self-testing kits in Zambia: a clusterrandomised trial nested in four HPTN 071 (PopART) intervention communities. Lancet HIV 2019;6:e81-92.

13 National Statistical Office (NSO) [Malawi] and ICF. Malawi demographic and health survey (DHS) 2015-16. Zomba, Malawi, and Rockville, Maryland, USA: NSO and ICF, 2017.

14 Neuman $\mathrm{M}$, Indravudh $\mathrm{P}$, Chilongosi R, et al. The effectiveness and cost-effectiveness of community-based lay distribution of HIV self-tests in increasing uptake of HIV testing among adults in rural Malawi and rural and peri-urban Zambia: protocol for StAR (self-testing for Africa) cluster randomized evaluations. BMC Public Health 2018;18:1234-34.

15 Hayes RJ, Moulton LH. Cluster randomised trials. Portland, OR: Taylor \& Francis Group, LLC, 2017.

16 Simwinga M, Kumwenda MK, Dacombe RJ, et al. Ability to understand and correctly follow HIV Self-Test kit Instructions for use: applying the cognitive interview technique in Malawi and Zambia. $J$ Int AIDS Soc 2019;22 Suppl 1:e25253.

17 Kumwenda MK, Johnson CC, Choko AT, et al. Exploring social harms during distribution of HIV self-testing kits using mixedmethods approaches in Malawi. J Int AIDS Soc 2019;22 Suppl 1:e25251-e51.

18 National Statistical Office (NSO) [Malawi] and ICF. Malawi demographic and health survey (DHS) 2010. Zomba, Malawi, and Rockville, Maryland, USA: NSO and ICF, 2011.

19 Sibanda E, Neuman M, Tumushime M. Linkage to care after HIV self-testing in Zimbabwe: a cluster-randomised trial. Conference on retroviruses and opportunistic infections (CROI 2018). Boston, Massachusetts, 2018.

20 Tsamwa D, Handima N, Sigande L. Does community-based distribution of HIV Self-Test kits increase uptake of HIV testing at population level? results of a cluster-randomised trial in Zambia. 3rd HIV research for prevention conference (R4P 2018). Madrid, Spain: International AIDS Society, 2018.

21 Choko AT, Corbett EL, Stallard N, et al. Hiv self-testing alone or with additional interventions, including financial incentives, and linkage to care or prevention among male partners of antenatal care clinic Attendees in Malawi: an adaptive multi-arm, multi-stage cluster randomised trial. PLoS Med 2019:16:e1002719.

22 Choko AT, Kumwenda MK, Johnson CC, et al. Acceptability of woman-delivered HIV self-testing to the male partner, and additional interventions: a qualitative study of antenatal care participants in Malawi. J Int AIDS Soc 2017;20:21610.

23 Dovel K, Shaba F, Offorjebe OA, et al. Effect of facility-based HIV self-testing on uptake of testing among outpatients in Malawi: a cluster-randomised trial. Lancet Glob Health 2020;8:e276-87.

24 Neuman M, Taegtmeyer M, Hatzold K, et al. Challenges in measurement of linkage following HIV self-testing: examples from the StAR project. J Int AIDS Soc 2019;22 Suppl 1:e25238-e38.

25 Cambiano V, Johnson CC, Hatzold K, et al. The impact and costeffectiveness of community-based HIV self-testing in sub-Saharan Africa: a health economic and modelling analysis. J Int AIDS Soc 2019;22 Suppl 1:e25243.

26 Mangenah C, Mwenge L, Sande L, et al. Economic cost analysis of door-to-door community-based distribution of HIV Self-Test kits in Malawi, Zambia and Zimbabwe. J Int AIDS Soc 2019;22 Suppl 1:e25255-e55

27 Mwenge L, Sande L, Mangenah C, et al. Costs of facilitybased HIV testing in Malawi, Zambia and Zimbabwe. PLoS One 2017;12:e0185740.

28 Indravudh PP, Fielding K, Kumwenda MK, et al. Community-led delivery of HIV self-testing to improve HIV testing, art initiation and broader social outcomes in rural Malawi: study protocol for a clusterrandomised trial. BMC Infect Dis 2019;19:814.

29 Bottomley C, Kirby MJ, Lindsay SW, et al. Can the buck always be passed to the highest level of clustering? BMC Med Res Methodol 2016;16:29 